\title{
Mixed linear/linear simplicial elements for incompressible elasticity and plasticity
}

\author{
M. Cervera ${ }^{\text {a,* }}$, M. Chiumenti ${ }^{\text {a }}$, Q. Valverde ${ }^{\text {b }}$, C. Agelet de Saracibar ${ }^{\text {a }}$ \\ ${ }^{a}$ International Center for Numerical Methods in Engineering (CIMNE), Universidad Politécnica de Cataluña (UPC), \\ Edificio C1, Campus Norte, Jordi Girona 1-3, 08034 Barcelona, Spain \\ b Departamento de Ingeniería, Sección Ingeniería Mecánica, Pontificia Universidad Católica del Perú, Lima, Peru
}

Received 11 December 2002; received in revised form 15 July 2003; accepted 23 July 2003

\begin{abstract}
This paper exploits the concept of orthogonal sub-grid scales to stabilize the behaviour of mixed linear/linear simplicial elements (triangles and tetrahedra) in incompressible or nearly incompressible situations. Both incompressible elastic and J2-plastic constitutive behaviours have been considered. The different assumptions and approximations used to derive the method are exposed. Implementation and computational aspects are also discussed, showing that a robust application of the proposed formulation is feasible. Numerical examples show that the elements derived are free of volumetric locking and spurious oscillations of the pressure, and that the results obtained compare favourably with those obtained with the $Q 1 P 0$ quadrilateral.
\end{abstract}

(C) 2003 Elsevier B.V. All rights reserved.

Keywords: Incompressibility; Plasticity; Stabilized finite element method; Orthogonal sub-grid scales

\section{Introduction}

Incompressible and nearly incompressible behaviour is often encountered in solid mechanics. Incompressible elasticity occurs in rubberlike materials and saturated porous solids in undrained conditions. Incompressible plasticity is observed in metals, which flow isochorically, following closely the von Mises model.

It is well known that standard irreductible low-order finite elements perform miserably in nearly incompressible situations, producing "solutions" which are almost completely "locked" by the incompressibility constraint. In fact, the purely incompressible problem does not admit an irreductible formulation and, consequently, a mixed framework in terms of displacements and pressure is necessary for these situations. Even though, many standard mixed finite elements, particularly those using low order

\footnotetext{
${ }^{*}$ Corresponding author.

E-mail address: miguel.cervera@ups.es (M. Cervera).
} 
interpolations, also perform poorly or totally fail to perform, producing results thoroughly polluted by spurious oscillations of the pressure. Over the years, and particularly in the 1990s, different strategies were suggested to reduce or avoid volumetric locking and pressure oscillations in finite element solutions [1-3]. Many modified mixed and enhanced formulations were proposed and tested to different degrees of success. Unfortunately, few approaches could be successfully applied to linear simplicial elements (triangles and tetrahedra), as shown for instance in [4] for the enhanced assumed strain method. This was due to the strictness of the B-B condition [5] when the standard Galerkin finite element method was applied straightforwardly to mixed low order elements, as it imposes severe restrictions on the compatibility of the interpolations used for the displacement and the pressure fields. One of these efforts was the "mini" element [6], an attractive linear triangle with a displacement interpolation enhanced with a cubic bubble function; even if it satisfies the B-B condition, it is only marginally stable, and it does not perform very well in many practical situations. However, there is great practical interest in using this sort of simple elements, mainly motivated by the fact that, now-a-days, tetrahedral meshes are relatively easy to generate for real life complex geometries. Therefore, this is today a very active area for research in solid mechanics [7-16].

In parallel, incompressible fluid dynamics has always been in the front line of research because of the innumerable practical applications of the field. In [17,18], the sub-grid scale approach was proposed as a new way of circumventing the difficulties posed by the B-B condition. The reasoning behind was not new, as it consisted of modifying the discrete variational form to attain control on the pressure field. The result was the possibility of using equal order interpolations and to construct stable low-order elements. Since then, the sub-grid concept has been extensively and fruitfully used in fluid dynamics. In [19,20], the concept

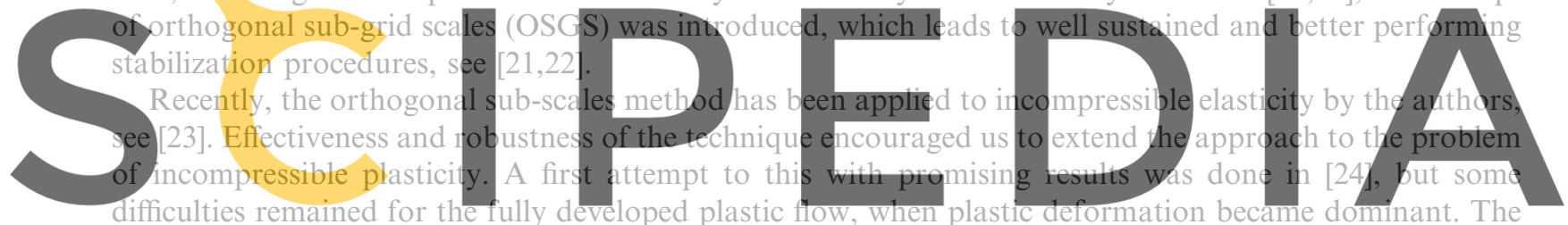

objective of the present work is to gain physical insight in order to introduce more realistic assumptions and

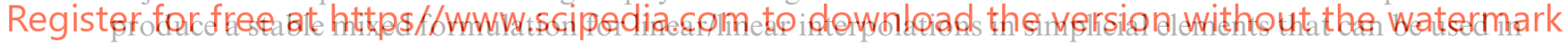

the whole incompressible elastic and plastic regimes.

The outline of the paper is as follows. In the next section the mixed displacement/pressure $(\mathbb{u} / p)$ finite element formulation for incompressible elasto-plastic behavior is summarized. Later, the sub-grid scale approach is outlined and a stabilization procedure, based in the idea of OSGS is presented. Implementation and computational aspects are discussed next. Finally, some numerical benchmarks and examples are presented to assess the present formulation and to compare its performance with the standard Galerkin elements as well as the well-known mixed Q1P0 quadrilateral.

\section{Mixed formulation for incompressible elasticity and plasticity}

The formulation of the incompressible elasto-plastic mechanical problem can be written considering the hydrostatic pressure $p$ as an independent unknown, additional to the primary displacement field. The stress tensor $\sigma$ can then be expressed as

$$
\boldsymbol{\sigma}=p \mathbf{1}+\mathbf{s}
$$

where $p=\frac{1}{3} \operatorname{tr}(\boldsymbol{\sigma})$ and $\mathbf{s}=\operatorname{dev}(\boldsymbol{\sigma})$ are the volumetric and the deviatoric parts of the stress tensor, respectively. Correspondingly, the strain tensor $\boldsymbol{\varepsilon}=\nabla^{\mathrm{s}} \mathbf{u}$, where $\mathbf{u}$ are the displacements, can be expressed as

$$
\boldsymbol{\varepsilon}(\mathbf{u})=\frac{1}{3} \varepsilon_{v} \mathbf{1}+\mathbf{e}
$$


where $\varepsilon_{\mathrm{v}}=\operatorname{tr} \boldsymbol{\varepsilon}=\nabla \cdot \mathbf{u}$ and $\mathbf{e}=\operatorname{dev} \boldsymbol{\varepsilon}$ are the volumetric and the deviatoric parts of the strain tensor, respectively. On the other hand, the constitutive equations can be expressed as

$$
\begin{aligned}
& p=K \varepsilon_{\mathrm{v}}^{\mathrm{e}}, \\
& \mathbf{s}=2 G \operatorname{dev} \varepsilon^{\mathrm{e}}=2 G \mathbf{e}^{\mathrm{e}},
\end{aligned}
$$

where $\varepsilon_{\mathrm{v}}^{\mathrm{e}}$ and $\mathrm{e}^{\mathrm{e}}$ are the elastic volumetric and the deviatoric parts of the strain tensor, respectively; $K$ is the bulk modulus, also referred to as modulus of volumetric compressibility, and $G$ is the shear modulus. In incompressible elasticity, $K$ tends to infinity and, thus, $\varepsilon_{\mathrm{v}}^{\mathrm{e}}$ vanishes. Additionally, in incompressible (J2) plasticity, the volumetric part of plastic deformation is zero, so that $\varepsilon_{\mathrm{v}}=\varepsilon_{\mathrm{v}}^{\mathrm{e}}=\nabla \cdot \mathbf{u}=0$.

With these definitions, the strong form of the continuum incompressible problem can be stated as: find the displacement field $\mathbf{u}$ and the pressure field $p$, for given prescribed body forces $\mathbf{f}$, such that

$$
\begin{aligned}
& \nabla \cdot \mathbf{s}+\nabla p+\mathbf{f}=0 \quad \text { in } \Omega, \\
& \nabla \cdot \mathbf{u}=0 \quad \text { in } \Omega,
\end{aligned}
$$

where $\Omega$ is the open and bounded domain of $\mathbb{R}^{n_{\mathrm{dim}}}$ occupied by the incompressible elasto-plastic solid in a space of $n_{\text {dim }}$ dimensions. Eqs. (5) and (6) are subjected to appropriate Diritchlet and Neumann boundary conditions. In the following, we will assume these, without loss of generality, in the form of prescribed displacements $\mathbf{u}=0$ on $\partial \Omega_{u}$, and prescribed tractions $\overline{\boldsymbol{t}}$ on $\partial \Omega_{t}$, respectively. In the mixed formulation the value of the pressure is defined by the Neumann conditions or, alternatively, by prescribing its value at

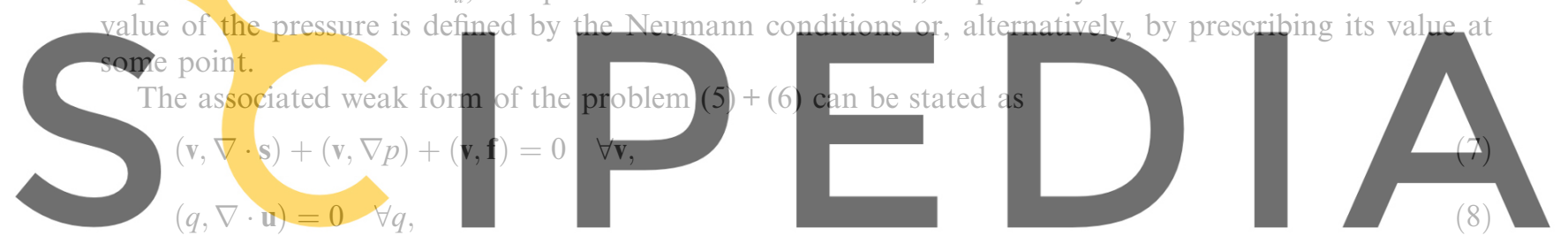

where $\mathbf{v} \in \mathscr{V}$ and $q \in \mathcal{Z}$ are the variations of the displacements and pressure fields, respectively, and $(\cdot$, .

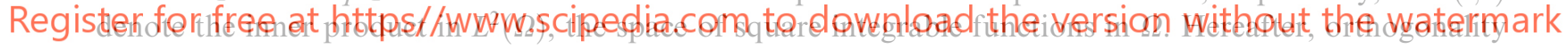

will be understood with respect to this product.

Integrating Eq. (7) by parts, the problem can be rewritten in the standard form as

$$
\begin{aligned}
& \left(\nabla^{\mathrm{s}} \mathbf{v}, \mathbf{s}\right)+(\nabla \cdot \mathbf{v}, p)-(\mathbf{v}, \mathbf{f})-(\mathbf{v}, \overline{\mathbf{t}})_{\partial \Omega}=0 \quad \forall \mathbf{v}, \\
& (q, \nabla \cdot \mathbf{u})=0 \quad \forall q .
\end{aligned}
$$

Observe that (9) and (10) involve the first derivative of the displacements and only the primal function of the pressure field. Hence, the natural spaces for displacements continuum fields and their variations $\mathbf{u}, \mathbf{v} \in \mathscr{V}=H_{0}^{1}(\Omega)$ are spaces of continuous functions with discontinuous derivatives, while pressure and its variation $p, q \in \mathscr{Q}=L^{2}(\Omega)$ can be sought in spaces including even discontinuous functions.

Let us now define the discrete finite element counterpart problem as

$$
\begin{aligned}
& \left(\nabla^{\mathrm{s}} \mathbf{v}_{\mathrm{h}}, \mathbf{s}_{\mathrm{h}}\right)+\left(\nabla \cdot \mathbf{v}_{\mathrm{h}}, p_{\mathrm{h}}\right)-\left(\mathbf{v}_{\mathrm{h}}, \mathbf{f}\right)-\left(\mathbf{v}_{\mathrm{h}}, \overline{\mathbf{t}}\right)_{\partial \Omega}=0 \quad \forall \mathbf{v}_{\mathrm{h}}, \\
& \left(q_{\mathrm{h}}, \nabla \cdot \mathbf{u}_{\mathrm{h}}\right)=0 \quad \forall q_{\mathrm{h}},
\end{aligned}
$$

where $\mathbf{u}_{\mathrm{h}}, \mathbf{v}_{\mathrm{h}} \in \mathscr{V}_{\mathrm{h}}$ and $p_{\mathrm{h}}, q_{\mathrm{h}} \in \mathscr{2}_{\mathrm{h}}$ are the discrete displacement and pressure fields and their variations, defined onto the finite element spaces $\mathscr{V}_{\mathrm{h}}$ and $\mathscr{Q}_{\mathrm{h}}$, respectively.

As it is well known, the stability of the discrete formulation depends on appropriate compatibility restrictions on the choice of the finite element spaces $\mathscr{V}_{\mathrm{h}}$ and $\mathscr{Q}_{\mathrm{h}}$, as stated by the BB-condition, [5]. According to this, standard Galerkin mixed elements with continuous equal order linear/linear interpolation for both 
fields are not stable, whereas stable mixed finite elements can be constructed using quadratic interpolation for the displacement field and constant interpolation for the pressure. Lack of stability shows as uncontrollable oscillations in the pressure field that entirely pollute the solution. Fortunately, the strictness of the B-B condition can be avoided by modifying the discrete variational form, for instance, by means of introducing appropriate numerical techniques that can provide the necessary stability to the desired choice of interpolation spaces. The objective of this work is to present a stabilization method, based in the orthogonal sub-grid scale approach, which allows the use of linear/linear interpolations for displacements and pressure.

\section{The sub-grid scale approach}

The basic idea of the sub-grid scale approach [171 is to consider that the continuous displacement field can be split in two components, one coarse and a finer one, corresponding to different scales or levels of resolution. The solution of the continuous problem contains components from both scales. For the solution of the discrete problem to be stable it is necessary to, somehow, include the effect of both scales in the approximation. The coarse scale can be appropriately solved by a standard finite element interpolation, which, however, cannot solve the finer scale. Nevertheless, the effect of this finer scale can be included, at least locally, to enhance the stability of the pressure in the mixed formulation.

To this end, the displacement field of the mixed problem will be approximated as

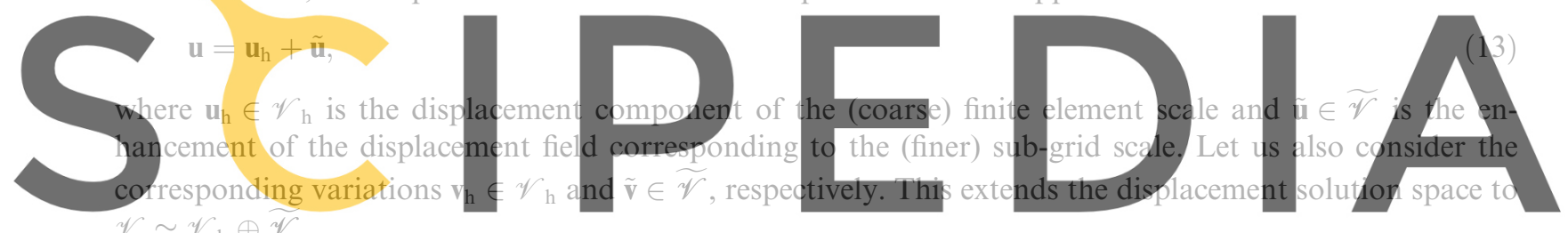

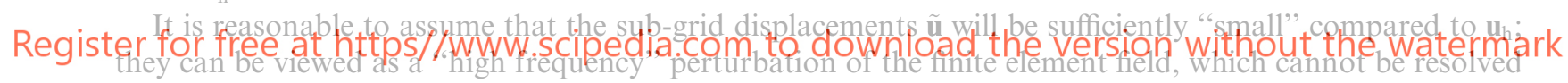

in $\mathscr{V}_{\mathrm{h}}$. It can also be assumed that $\tilde{\mathbf{u}}$ and $\tilde{\mathbf{v}}$ vanish on the boundary $\partial \Omega$. It must be pointed out that no subgrid scale contribution has been considered on the pressure field.

Considering the sub-scales, the deviatoric stresses can be decomposed into two different contributions

$$
\mathbf{s}(\mathbf{u})=\mathbf{s}_{\mathrm{h}}(\mathbf{u})+\tilde{\mathbf{s}}(\mathbf{u}) \text {. }
$$

Notice that the stresses $\mathbf{s}$ are not a linear function of $\mathbf{u}$ and, therefore, it is not exactly true that $\mathbf{s}_{\mathrm{h}}=\mathbf{s}_{\mathrm{h}}\left(\mathbf{u}_{\mathrm{h}}\right)$ and $\tilde{\mathbf{s}}=\tilde{\mathbf{s}}(\tilde{\mathbf{u}})$. However, we can approximate the deviatonic stresses corresponding to the coarse finite element scale in the standard fashion, as

$$
\mathbf{s}_{\mathrm{h}}(\mathbf{u}) \cong \mathbf{s}_{\mathrm{h}}\left(\mathbf{u}_{\mathrm{h}}\right)=2 G \mathbf{e}_{\mathrm{h}}^{\mathrm{e}}
$$

which can be computed following any of the established algorithms for elasto-plasticity. Let us now introduce the secant shear modulus as (half) the ratio between the norms of the deviatoric stress and total strain tensors, $2 G^{*}=\left\|\mathbf{s}_{\mathrm{h}}\right\| /\left\|\mathbf{e}_{\mathrm{h}}\right\|$. For plasticity, this ratio is obviously non-constant and it varies along the deformation process.

Being the enhancement $\tilde{\mathbf{u}}$ "small" compared to $\mathbf{u}_{\mathrm{h}}$, it is possible to make the following approximation for the strains due to the sub-scale:

$$
\frac{\|\tilde{\mathbf{s}}\|}{\|\tilde{\mathbf{e}}\|} \cong \frac{\left\|\mathbf{s}_{\mathrm{h}}\right\|}{\left\|\mathbf{e}_{\mathrm{h}}\right\|}=2 G^{*},
$$


which motivates the approximation

$$
\tilde{\mathbf{s}}(\mathbf{u}) \cong \tilde{\mathbf{s}}(\tilde{\mathbf{u}})=2 G^{*} \tilde{\mathbf{e}} .
$$

To assume that the split into elastic and plastic strains is the same for both coarse and fine scales, Eq. (16), is a refinement on the assumption used in [24], where it was supposed that sub-grid scale induces no further plastic strains and, thus, $\tilde{\mathbf{s}} \cong 2 G \tilde{\mathbf{e}}$. This new hypothesis is more realistic once the plastic part of the deformation grows significantly.

It can be remarked that the formalism of approximation (17) holds for other non-linear constitutive models, such as nonlinear elasticity or continuum damage mechanics. It is expected that the present stabilization procedure can also be applied to those models.

With these definitions, the discrete problem corresponding to Eqs. (7) and (8) is now

$$
\begin{aligned}
& \left(\nabla^{\mathrm{s}} \mathbf{v}_{\mathrm{h}}, \mathbf{s}_{\mathrm{h}}\right)+\left(\nabla^{\mathrm{s}} \mathbf{v}_{\mathrm{h}}, \tilde{\mathbf{s}}\right)+\left(\nabla \cdot \mathbf{v}_{\mathrm{h}}, p_{\mathrm{h}}\right)-\left(\mathbf{v}_{\mathrm{h}}, \mathbf{f}\right)-\left(\mathbf{v}_{\mathrm{h}}, \overline{\mathbf{t}}\right)_{\partial \Omega_{t}}=0 \quad \forall \mathbf{v}_{\mathrm{h}}, \\
& \left(\tilde{\mathbf{v}}, \nabla \cdot \mathrm{s}_{\mathrm{h}}\right)+(\tilde{\mathbf{v}}, \nabla \cdot \widetilde{\mathbf{s}})+\left(\widetilde{\mathbf{v}}, \nabla p_{\mathrm{h}}\right)+(\widetilde{\mathbf{v}}, \mathbf{f})=0 \quad \forall \widetilde{\mathbf{v}}, \\
& \left(q_{\mathrm{h}}, \nabla \cdot \mathbf{u}_{\mathrm{h}}\right)+\left(q_{\mathrm{h}}, \nabla \cdot \widetilde{\mathbf{u}}\right)=0 \quad \forall q_{\mathrm{h}},
\end{aligned}
$$

where Eq. (18) has been integrated by parts, recalling that $\tilde{\mathbf{u}}$ and $\tilde{\mathbf{v}}$ vanish on the boundary.

Due to the approximation used, Eq. (13), and the linear independence of $\mathbf{v}_{\mathrm{h}}$ and $\tilde{\mathbf{v}}$, now the continuum equation (10) unfolds in two discrete equations, one related to each scale considered. Eqs. (18) and (20) are defined in the finite element spaces $\mathscr{V}_{\mathrm{h}}$ and $\mathscr{2}_{\mathrm{h}}$, respectively. The first one solves the balance of momentum incleding a stabilization term the incompressibility conditio displacements ü. On the other bo solved by the finite elemen $-(\tilde{\mathbf{v}}, \nabla \cdot \tilde{\mathbf{s}})=\left(\widetilde{\mathbf{v}}, \mathbf{r}_{\mathrm{h}}\right)$
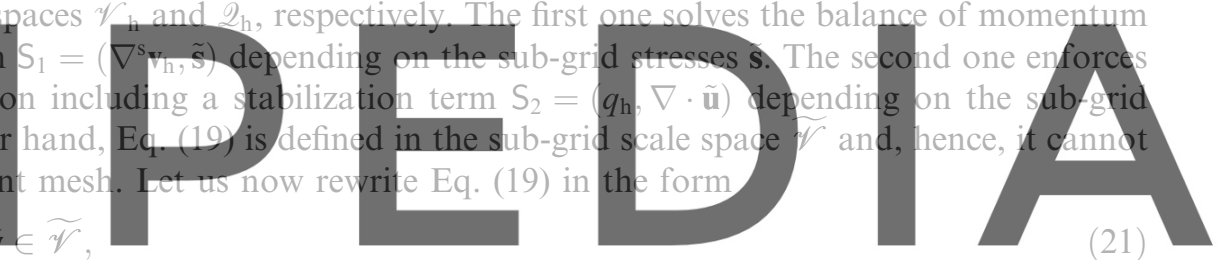

where the residual of the Cauchy equation in the finite element scale is defined as

Register for free at https//WWW.scipedia.com to download the version without the watermark $\mathrm{r}_{\mathrm{h}}=\mathbb{r}_{\mathrm{h}}\left(\mathbf{u}_{\mathrm{h}}, p_{\mathrm{h}}\right)=\nabla \cdot \mathrm{s}_{\mathrm{h}}+\nabla p_{\mathrm{h}}+\mathbf{f}$.

Now, some appropriate approximations are necessary. According to hypotheses (16) and (17), let us assume that, within each finite element $\Omega_{e}$

$$
\|\nabla \cdot \tilde{\mathbf{s}}\| \cong \frac{\|\tilde{\mathbf{s}}\|}{c_{1} h_{e}}=\frac{2 G_{e}^{*}\|\tilde{\mathbf{e}}\|}{c_{1} h_{e}} \cong \frac{2 G_{e}^{*}}{c_{1} h_{e}}\left(\frac{\|\tilde{\mathbf{u}}\|}{c_{2} h_{e}}\right)=\frac{1}{\tau_{e}}\|\tilde{\mathbf{u}}\|,
$$

where the parameter $\tau_{e}=c h_{e}^{2} / 2 G_{e}^{*}$ is defined as a function of the characteristic length of the element $h_{e}$ and the current secant shear modulus $G_{e}^{*}$. The constant $c=c_{1} c_{2}=\mathcal{O}(1)$ has to be determined through numerical testing. This very simple and heuristic approximation of the effect of the sub-scale is one of the keys to the success of the stabilization procedure of the elasto-plastic incompressible problem. More elaborated assumptions may be needed in other situations.

Integrating the stabilization terms by parts, and recalling that the sub-scale displacements vanish on the boundary, it yields

$$
\begin{aligned}
& \mathrm{S}_{1}=\left(\nabla^{\mathrm{s}} \mathbf{v}_{\mathrm{h}}, \tilde{\mathbf{s}}\right)=-\left(\tilde{\mathbf{u}}, \nabla \cdot\left(2 G^{*} \operatorname{dev} \nabla^{\mathrm{s}} \mathbf{v}_{\mathrm{h}}\right)\right), \\
& \mathrm{S}_{2}=\left(q_{\mathrm{h}}, \nabla \cdot \tilde{\mathbf{u}}\right)=-\left(\tilde{\mathbf{u}}, \nabla q_{\mathrm{h}}\right) .
\end{aligned}
$$

The term $\nabla \cdot\left(\operatorname{dev} \nabla^{\mathrm{s}} \mathbf{v}_{\mathrm{h}}\right)$ in (24) involves second derivatives of finite element functions which vanish when linear elements are used. In the case of higher order elements these derivatives can be neglected, leading to a method which is still consistent, but with a non-optimal rate of convergence [22]. 


\section{Orthogonal sub-grid scales}

The objective in this section is to obtain a useful expression for the sub-grid scale displacements $\tilde{\mathbf{u}}$ to be introduced in the stabilization term $S_{2}$ in Eq. (20). It was argued in [20] that a very natural choice for the unknown sub-grid space $\widetilde{\mathscr{V}}_{\mathrm{h}}$ is the space orthogonal to the finite element space, referred to hereafter as $\mathscr{V}_{\mathrm{h}}^{\perp}$. This means approximating the displacement solution space as $\mathscr{V} \simeq \mathscr{V}_{\mathrm{h}} \oplus \mathscr{V}_{\mathrm{h}}^{\perp}$. The subsequent stabilization method is called orthogonal sub-grid scale method, and it has already been successfully applied to several problems in fluid mechanics.

Also, the format of the Eq. (21), which is exact for the fine scale and nonlocal, strongly suggests that $\nabla \cdot \tilde{\mathbf{s}}$, and hence $\tilde{\mathbf{u}}$, are driven by the residual of the coarse scale, $\mathbf{r}_{\mathrm{h}}$. In [18] it is reasoned that the sought effect of the finer scale is to explicitly account for the distributional effects of the residual of the coarse scale.

Because of these two reasons, we will take $\tilde{\mathbf{v}} \in \mathscr{V}_{\mathrm{h}}^{\perp}$, and assume that

$$
-(\tilde{\mathbf{v}}, \nabla \cdot \tilde{\mathbf{s}}) \cong\left(\tilde{\mathbf{v}}, P_{\mathrm{h}}^{\perp}\left(\mathrm{r}_{\mathrm{h}}\right)\right) \quad \forall \tilde{\mathbf{v}} \in \mathscr{V}_{\mathrm{h}}^{\frac{1}{\mathrm{~h}}},
$$

where $P_{\mathrm{h}}^{\perp}(\mathbf{x})$ is the orthogonal projection of $\mathbf{x}$ onto $\mathscr{V}_{\mathrm{h}}^{\perp}$, which can be expressed as $P_{\mathrm{h}}^{\perp}(\mathbf{x})=\mathbf{x}-P_{\mathrm{h}}(\mathbf{x})$. The $L^{2}$ projection of $\mathbf{x}$ onto the finite element space, or least square fitting, can be computed from the orthogonality condition

$$
\left(P_{\mathrm{h}}(\mathbf{x})-\mathbf{x}, \boldsymbol{\eta}_{\mathrm{h}}\right)=0 \quad \forall \boldsymbol{\eta}_{\mathrm{h}} \in \mathscr{V}_{\mathrm{h}} .
$$

Using approximations (23) and (26), the sub-scale displacements can be localized within each finite ele-

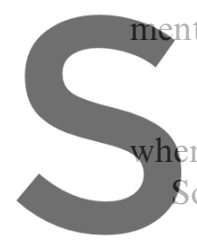
$\Omega_{e}$, and be expressec
$\tilde{\mathrm{u}}_{e}$
$\tilde{\mathbf{u}}_{e}=\tau_{e} P_{\mathrm{h}}^{\perp}\left(\mathrm{r}_{\mathrm{h}}\right) \in \mathscr{V} \frac{\perp}{\mathrm{h}}$

re the positive sign is necessary
ome remarks are in order:
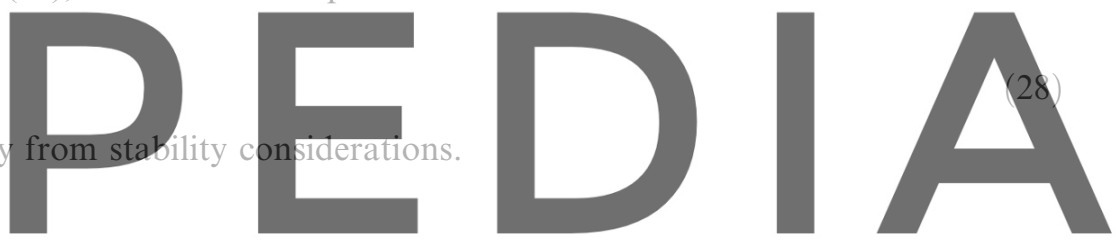

. As expected, $\widetilde{\mathbf{u}}$ is sufficiently "small" compared to $\mathbf{u}_{\mathrm{h}}\left(\widetilde{\mathbf{u}} \simeq \mathcal{O}\left(h^{2}\right)\right)$.

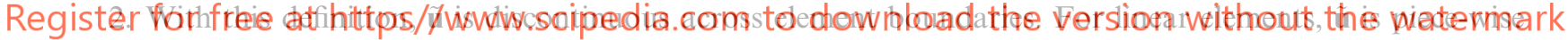
linear.

3. Even if defined element-wise, $\tilde{u}_{e}$ cannot be condensed at element level, because $P_{\mathrm{h}}^{\perp}(\cdot)$ is a global operator.

4. In the localization process, it is necessary to neglect the integrals over element faces involving the subscale, in front of the integrals over the element volumes. This is justified in [22] resorting to Fourier analysis and recalling that the sub-scale is associated to higher frequencies that the grid scale. It is worth to mention that for "bubble"-type enhancements these boundary terms are null by construction [25,26].

5. Eq. (28) does not need to be interpreted point-wise, as the values of $\tilde{\mathbf{u}}$ are not used in the stabilization procedure; only the integrals $S_{1}$ and $S_{2}$ in Eqs. (24) and (25) are needed.

It must be pointed out that $\mathbf{f}$ in (22) can be assumed to belong to the space $\mathscr{V}_{\mathrm{h}}$ and, consequently, $P_{\mathrm{h}}^{\perp}(\mathbf{f})=\mathbf{0}$. Also, $\nabla \cdot \mathbf{s}_{\mathrm{h}}$ in (22) involves second derivatives of finite element functions which vanish when linear elements are used. Taking all this into account, expression (28) transforms in

$$
\tilde{\mathbf{u}}_{e}=\tau_{e}\left(\nabla p_{\mathrm{h}}-P_{\mathrm{h}}\left(\nabla p_{\mathrm{h}}\right)\right) \text {. }
$$

Finally, substituting Eq. (29) into the expression of the stabilization term $S_{2}$, see Eq. (25), it simplifies as

$$
\mathrm{S}_{2}=-\sum_{e=1}^{n_{\mathrm{elm}}} \tau_{e}\left(\nabla q_{\mathrm{h}} \cdot\left[\nabla p_{\mathrm{h}}-P_{\mathrm{h}}\left(\nabla p_{\mathrm{h}}\right)\right]\right) .
$$

Observe that this stabilization term is computed in an element by element manner and, within each element, its magnitude depends on the difference between the continuous (projected) and the discontinuous (ele- 
mental) pressure gradient. This means that the term added to secure a stable solution decreases very rapidly upon mesh refinement, as the finite element scale becomes finer and the projection of the residual reduces. This happens at a greater rate than with other stabilization techniques, such as the Galerkin Least Square (GLS) method, where the stabilization terms are proportional to the residual itself $[27,28]$.

The projection of the pressure gradient onto the finite element space $\mathscr{V}_{\mathrm{h}}, \Pi_{\mathrm{h}}=P_{\mathrm{h}}\left(\nabla p_{\mathrm{h}}\right)$, is computed as

$$
\left(\nabla p_{\mathrm{h}}, \boldsymbol{\eta}_{\mathrm{h}}\right)=\left(\boldsymbol{\Pi}_{\mathrm{h}}, \boldsymbol{\eta}_{\mathrm{h}}\right) \quad \forall \boldsymbol{\eta}_{\mathrm{h}} \in \mathscr{V}_{\mathrm{h}} .
$$

As a result of the above procedure, the stabilized mixed system of equations proposed in this work to solve the problem of incompressible elasto-plastic behavior with linear/linear interpolations for the displacement and pressure fields is the following:

$$
\begin{aligned}
& \left(\nabla^{\mathrm{s}} \mathbf{v}_{\mathrm{h}}, \mathbf{s}_{\mathrm{h}}\right)+\left(\nabla \cdot \mathbf{v}_{\mathrm{h}}, p_{\mathrm{h}}\right)-\left(\mathbf{v}_{\mathrm{h}}, \mathbf{f}\right)-\left(\mathbf{v}_{\mathrm{h}}, \overline{\mathbf{t}}\right)_{\partial \Omega_{t}}=0 \quad \forall \mathbf{v}_{\mathrm{h}}, \\
& \left(q_{\mathrm{h}}, \nabla \cdot \mathrm{u}_{\mathrm{h}}\right)-\sum_{e=1}^{n_{\mathrm{elm}}} \tau_{e}\left(\nabla q_{\mathrm{h}} \cdot\left[\nabla p_{\mathrm{h}}-\Pi_{\mathrm{h}}\right]\right)=0 \quad \forall q_{\mathrm{h}}, \\
& \left(\nabla p_{\mathrm{h}}, \boldsymbol{\eta}_{\mathrm{h}}\right)-\left(\Pi_{\mathrm{h}}, \eta_{\mathrm{h}}\right)=0 \quad \forall \eta_{\mathrm{h}} .
\end{aligned}
$$

It is important to point out that under these hypotheses no stabilization term appears in the first equation (32), which can be solved as in a standard mixed finite element formulation. The only remaining stabilization term appears in the incompressibility equation (33). Finally, it must be observed that in this formulation there is a third nodal variable $\mathbf{H}_{\mathrm{h}}$ involved. However, in the next section it is shown that this drawback can be overcome to 5. Implementation and computationa

Due to the nonlinear dependence of the stresses on the displacements, the solution of the system of equations (32)-(34) requires the use of an appropriate incremental/iterative procedure such as the Newton-

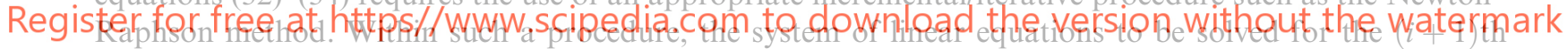

equilibrium iteration of the $(n+1)$ th time (or load) step is

$$
\left[\begin{array}{ccc}
\mathbf{K}_{\mathrm{dev}}^{(n+1, i)} & \mathbf{G} & \mathbf{0} \\
\mathbf{G}^{\mathrm{T}} & -\mathbf{L}_{\tau} & \mathbf{G}_{\tau}^{\mathrm{T}} \\
\mathbf{0} & \mathbf{G}_{\boldsymbol{\tau}} & -\mathbf{M}_{\tau}
\end{array}\right]\left[\begin{array}{l}
\delta \mathbf{U} \\
\delta \mathbf{P} \\
\delta \boldsymbol{\Pi}
\end{array}\right]^{(n+1, i+1)}=-\left[\begin{array}{c}
\mathbf{R}_{1} \\
\mathbf{R}_{2} \\
\mathbf{0}
\end{array}\right]^{(n+1, i)}
$$

where $\delta \mathbf{U}, \delta \mathbf{P}$ and $\delta \boldsymbol{\Pi}$ are the iterative corrections to the nodal values for the displacements, pressure and pressure gradient, respectively, $\mathbf{R}_{1}$ and $\mathbf{R}_{2}$ are the residual vectors associated to the satisfaction of the balance of momentum and incompressibility equations, respectively, and the global matrices $\mathbf{K}_{\mathrm{dev}}^{(n+1, i)}, \mathbf{G}, \mathbf{G}_{\tau}$, $\mathbf{L}_{\tau}$ and $\mathbf{M}_{\tau}$ come from the standard assembly procedure of the elemental contributions. This global matrix is symmetric, but it is not positive definite. Each one of the elemental matrices $\mathbf{K}^{(\mathrm{e})}$ has the symmetric structure

$$
\left[\mathbf{K}^{A B}\right]^{(\mathrm{e})}=\left[\begin{array}{ccc}
\mathbf{K}_{\mathrm{dev}}^{A B} & \mathbf{G}^{A B} & \mathbf{0} \\
\left(\mathbf{G}^{A B}\right)^{\mathrm{T}} & -\tau_{e} L^{A B} & \tau_{e}\left(\mathbf{G}^{A B}\right)^{\mathrm{T}} \\
\mathbf{0} & \tau_{e} \mathbf{G}^{A B} & -\tau_{e} \mathbf{M}^{A B}
\end{array}\right]^{(\mathrm{e})},
$$

where the entry $(\cdot)^{A B}$ is a sub-matrix corresponding to the local nodes $A$ and $B$. In Eq. (36), $\mathbf{K}_{\text {dev }}^{A B}$ is the deviatoric component of the standard elasto-plastic tangent stiffness matrix defined as

$$
\mathbf{K}_{\mathrm{dev}}^{A B}=\int_{\Omega_{e}} \mathbf{B}_{A}^{\mathrm{T}} \mathbf{D}_{\mathrm{dev}}^{\mathrm{ep}} \mathbf{B}_{B} \mathrm{~d} \Omega
$$


where $\mathbf{D}_{\mathrm{dev}}^{\mathrm{ep}}$ is the deviatoric constitutive matrix and $\mathbf{B}$ is a standard deformation sub-matrix. The generic term of the discrete gradient matrix operator $\mathbf{G}^{A B}$ is given by

$$
\mathbf{G}^{A B}=\int_{\Omega_{e}}\left[\nabla N^{A}\right] N^{B} \mathrm{~d} \Omega, \quad \text { where }\left[\nabla N^{A}\right]=\left[\begin{array}{lll}
N_{, x}^{A} & N_{, y}^{A} & N_{, z}^{A}
\end{array}\right]^{\mathrm{T}},
$$

while the Laplacian term $L^{A B}$ can be expressed as

$$
L^{A B}=\int_{\Omega_{e}}\left[\nabla N^{A}\right]^{\mathrm{T}}\left[\nabla N^{B}\right] \mathrm{d} \Omega .
$$

Finally, $\mathbf{M}^{A B}$ is the "mass" matrix associated to the displacement field

$$
\mathbf{M}^{A B}=\int_{\Omega_{e}} \mathbf{N}^{A} \mathbf{N}^{B} \mathrm{~d} \Omega .
$$

The monolithic solution of system (35) can be avoided by using a staggered procedure, in which the pressure projection $\boldsymbol{\Pi}^{(n+1, i+1)}$ is solved independently and explicitly. To this end, from the third equation, it is possible to express $\mathbf{\Pi}{ }^{(n+1, i+1)}$ in terms $\mathbf{P}^{(n+1, i+1)}$ as

$$
\boldsymbol{\Pi}^{(n+1, i+1)}=\mathbf{M}_{\tau}^{-1} \mathbf{G}_{\tau} \mathbf{P}^{(n+1, i+1)} \cong \overline{\mathbf{M}}^{-1} \mathbf{G} \mathbf{P}^{(n+1, i+1)} .
$$

The computation of the projections $\Pi$ can be transformed in a straight-forward operation by neglecting the difference in the $\tau_{e}$ coefficient in adjacent elements and considering an approximate lumped mass matrix $\overline{\mathbf{M}}$.

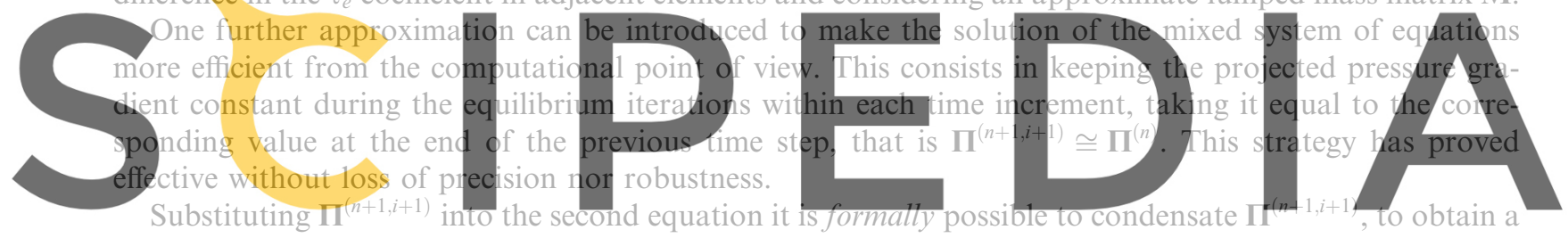

reduced system with the form

Register for free at https//www.scipedia.com to download the version without the watermark

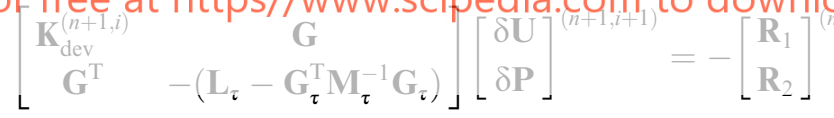

The elimination of the projection $\boldsymbol{\Pi}$ is not feasible in practice, because it is a continuous variable and, therefore, the condensation procedure cannot be performed at element level; if performed at global level it would yield a system reduced but with a totally spoiled banded structure. However, in this reduced form the overall effect of the proposed stabilization method becomes self-evident. It is interesting to note that it resembles the format of the enhanced assumed strain method, where the enhancing fields are discontinuous and their variables can be condensed at local level.

\section{Numerical results}

The formulation presented in the preceding sections is illustrated below in a number of benchmark problems. Performance of the stabilization method is tested considering 2D plane-strain triangular meshes and 3D tetrahedral meshes. All the examples involve incompressible elasticity and perfect $\mathbf{J} 2$-plasticity, but as the standard and $Q 1 P 0$ formulations cannot deal with the purely incompressible case, the following material properties are assumed: Young's modulus $E=10 \mathrm{MPa}$, Poisson's ratio $v=0.499$ (recall that $G=E / 2(1+v), K=E / 3(1-2 v))$, yield stress $\sigma_{\mathrm{Y}}=E / 1000$. Perfect plasticity is considered because it should allow collapse mechanics to develop neatly and limit loads to be clearly defined. A value $c=1$ is taken for the evaluation of $\tau_{e}$. 
The Newton-Raphson method, combined with a line search procedure is used to solve the non-linear system of equations arising from the spatial and temporal discretization of the weak form of the stabilized problem. In all cases 50 time (load) steps are performed to complete the analyses. Calculations are performed with an enhanced version of the finite element program COMET [29], developed by the authors at the International Center for Numerical Methods in Engineering (CIMNE). Pre- and post-processing is done with GiD [30], also developed at CIMNE.

\subsection{D vertical cut}

The first example is a plane-strain 2D vertical cut loaded through a rigid footing which sustains a central point load. Fig. 1a depicts the geometry of the problem; dimensions are related to length $a=5 \mathrm{~m}$. Fig. 1 also shows the meshes used in the analyses: (b) an unstructured mesh of 843 linear triangles (463 nodes), and (c) a structured $20 \times 20+20$ mesh of Q1P0 quadrilaterals (462 nodes). In the unstructured mesh, the pre-processor used tends to introduce patches or equilateral triangles with predominant directions at $-30^{\circ}$, $+30^{\circ}$ and $+90^{\circ}$,

Fig. 2 shows load versus (point of application)-displacement curves for the (a) standard irreductible P1, (b) standard mixed PIP1, (c) stable mixed PIPI-S and (d) QIPO cases (1 m thickness is assumed). It is remarkable that the last two achieve a well defined limit load, with practically overlapping curves; the standard mixed formulation obtains a slightly higher limit load, while the irreductible formulation clearly exhibits volumetric locking, both in the elastic and plastic regimes.

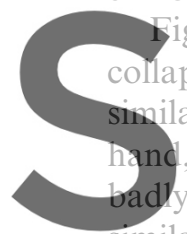

1ig. 3 depicts contours of collapse mechanism can b

similar results are obtained wit

hand, the standard irreductibit affected by the mesh

similar to the one developed by the stable elements.

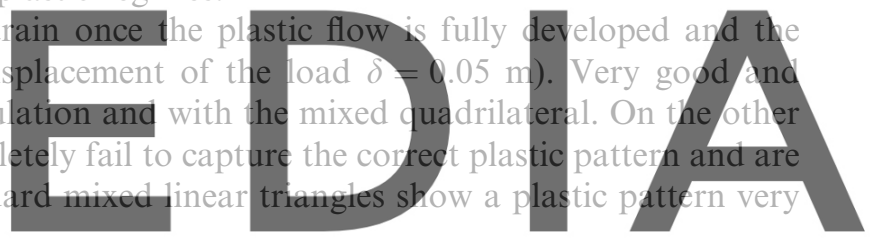

Fig. 4 presents pressure contours at the same time (final) of the deformation process. Lack of stability

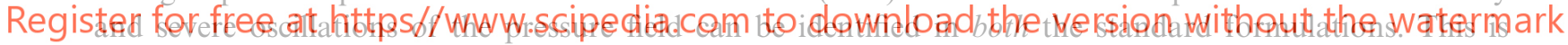

enough to completely destabilize the irreductible formulation, although for this example it seems to have little influence in the deformation pattern, as the plastic deformation does not depend on the pressure.

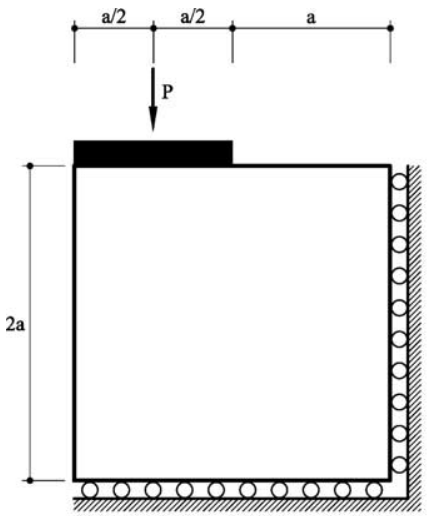

(a)

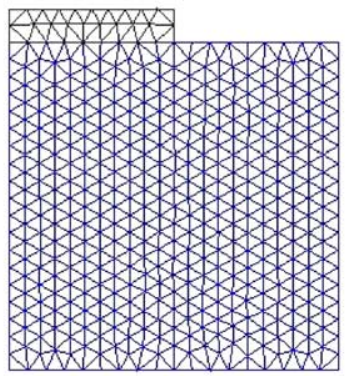

(b)

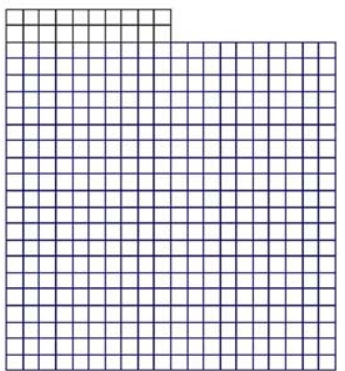

(c)

Fig. 1. Meshes for the 2D vertical cut: (a) geometry of the problem; (b) unstructured; (c) structured. 


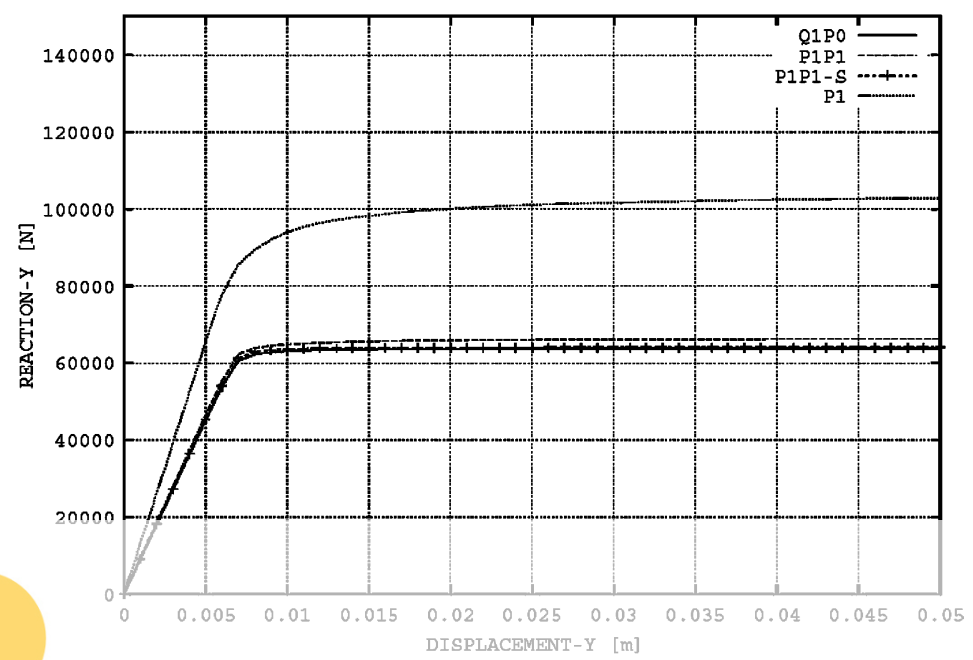

Fig. 2. Load-displacement curve for 2D vertical cut.
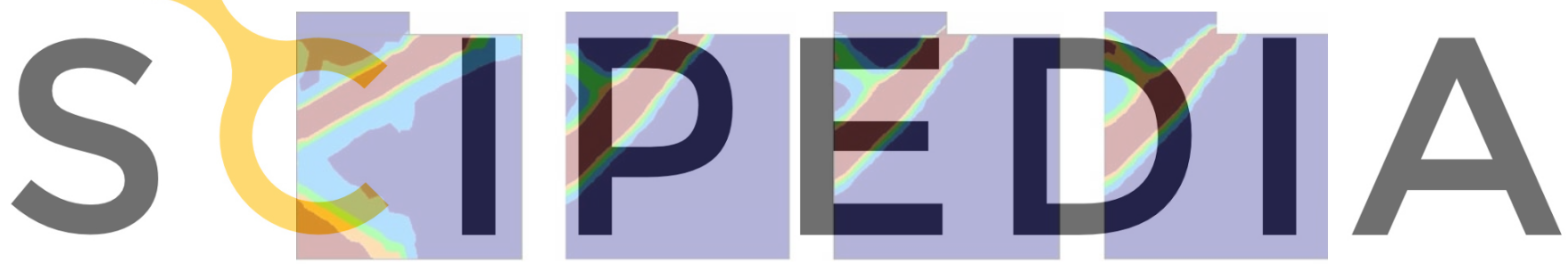

Register for free at https//www.scipedia.com to download the version without the watermark Fig. 3. Equivalent plastic strain contour fills for the 2D vertical cut with different formulations: (a) P1, (b) P1P1, (c) P1P1-S and (d) $Q 1 P 0$.

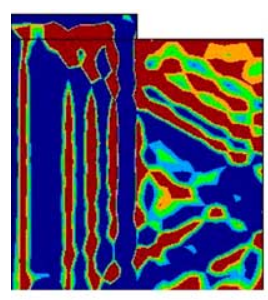

(a)

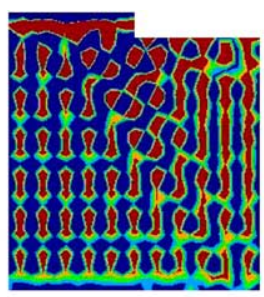

(b)

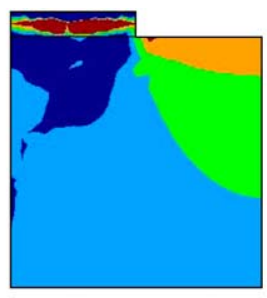

(c)

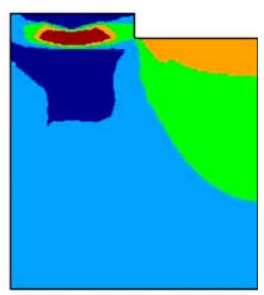

(d)

Fig. 4. Pressure contour fills for the $2 \mathrm{D}$ vertical cut with different formulations: (a) $P 1$, (b) $P 1 P 1$, (c) $P 1 P 1-S$, (d) $Q 1 P 0$.

Obviously, this is not always necessarily the case, and especially in nonlinear analyses. The improved performance of the proposed formulation is easily perceptible.

Concerning the CPU time required for the solution, the ratios between the four cases are 1.28/1.50/1.58/ 1.00 , taking the quadrilateral element as reference. The proposed formulation is $58 \%$ more costly than the quadrilateral (because of the 2/1 ratio for the number of elements and 3/2 ratio for the number of dofs), but the difference in cost with the standard mixed triangles (same number of elements and dofs) is very small. 


\section{2. $3 D$ vertical cut}

A $3 \mathrm{D}$ version of the previous problem is depicted in Fig. $5 \mathrm{~b}$; dimensions are related to length $a=5 \mathrm{~m}$. Fig. 5b shows the unstructured mesh of linear P1P1-S tetrahedra used in the computations (9533 nodes, 50,080 elements). A structured $20 \times 20 \times 20+200$ element mesh ( 9503 nodes), not shown, is used for comparing results with the $Q 1 P 0$ hexahedron.

Fig. 6 shows load versus (point of application)-displacement curves for both cases. Again, it is remarkable that both formulations achieve a well-defined limit load.

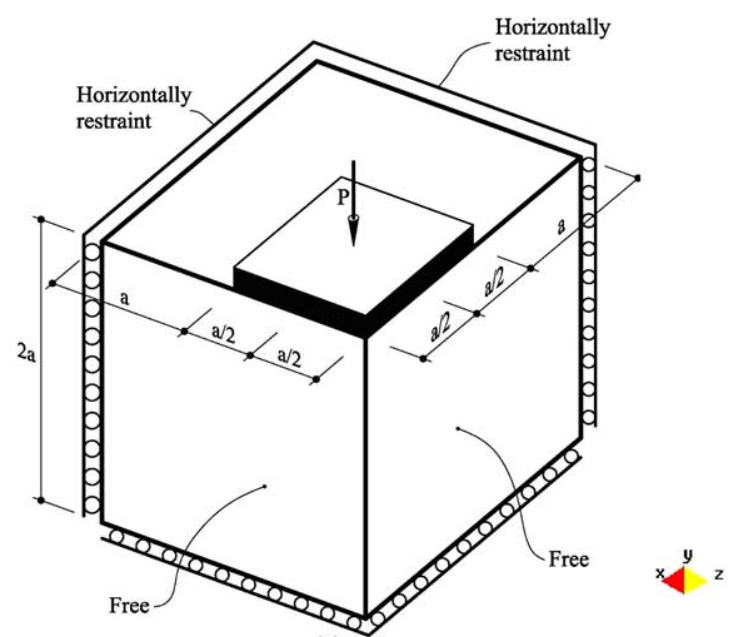

(a)

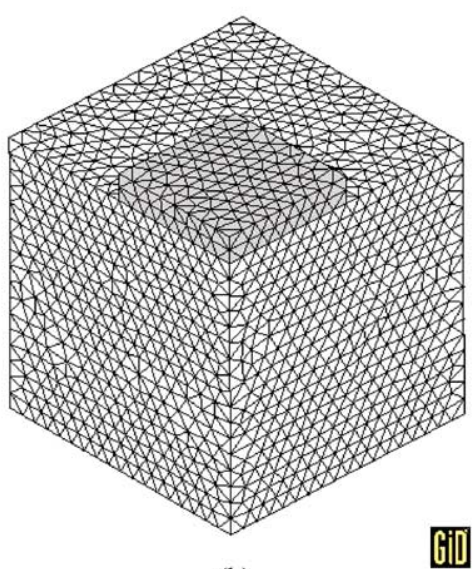

(b)

Fig. 5. Geometry and unstructured mesh for $3 \mathrm{D}$ vertical cut.

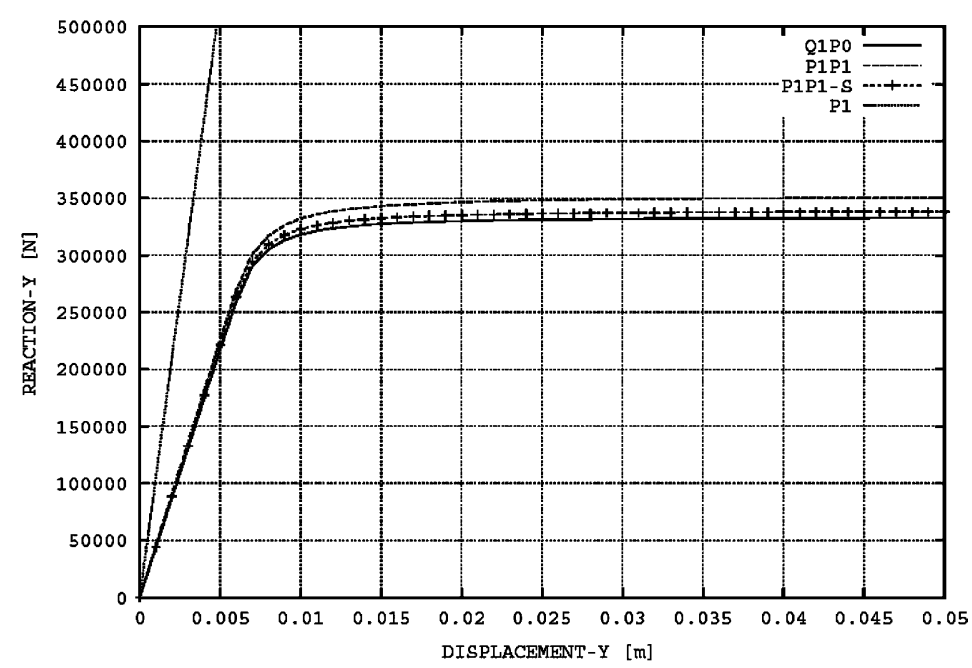

Fig. 6. Load-displacement curve for 3D vertical cut. 


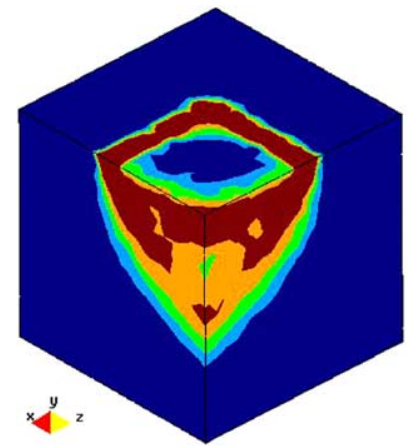

(a)

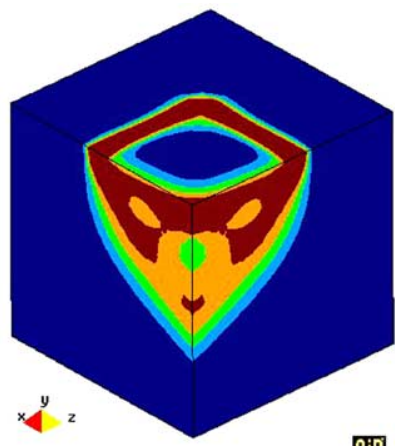

(b)

Fig. 7. Equivalent plastic strain contour fills for $3 \mathrm{D}$ vertical cut with different formulations: (a) P1P1-S, (b) $Q 1 P 0$.

Fig. 7 displays contours of the effective plastic strain once the plastic flow is fully developed (vertical displacement of the load $\delta=0.05 \mathrm{~m}$ ). Again, it is remarkable that satisfactory results are obtained with the proposed formulation in an unstructured mesh of tetrahedra, and very similar to those obtained in an structured and regular mesh of hexahedra.

The proposed formulation is 53\% more costly than the hexahedron (because of the $6 / 1$ ratio for the number of elements and the $4 / 3$ ratio for the number of dofs).

\subsection{D Prandtl's punch test}

The last example is the Prandtl's punch test, a well-known plane-strain 2D problem often used in the literature to test the ability of J2-plastic formulations to capture collapse loads and mechanisms. Fig. 8 depicts the geometry of the problem, again a rigid footing with a central point load; dimensions are related to length $b=1 \mathrm{~m}$. Because of the symmetry, only half of the domain needs to be discretized. Two meshes were used in the computations, an unstructured one with linear triangles and a structured one with quadrilaterals $(40 \times 40+40$ elements $)$, both with about 1,700 nodes.

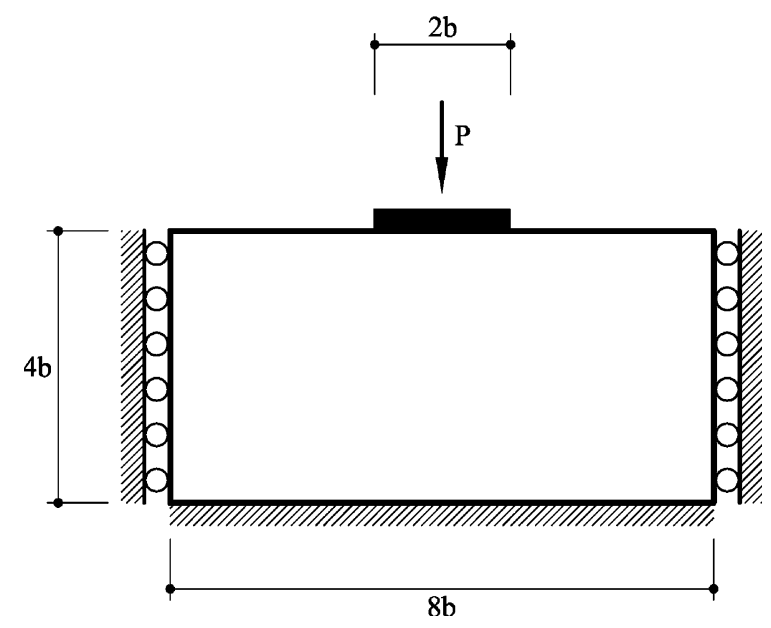

Fig. 8. Geometry for 2D Prandtl's punch test. 


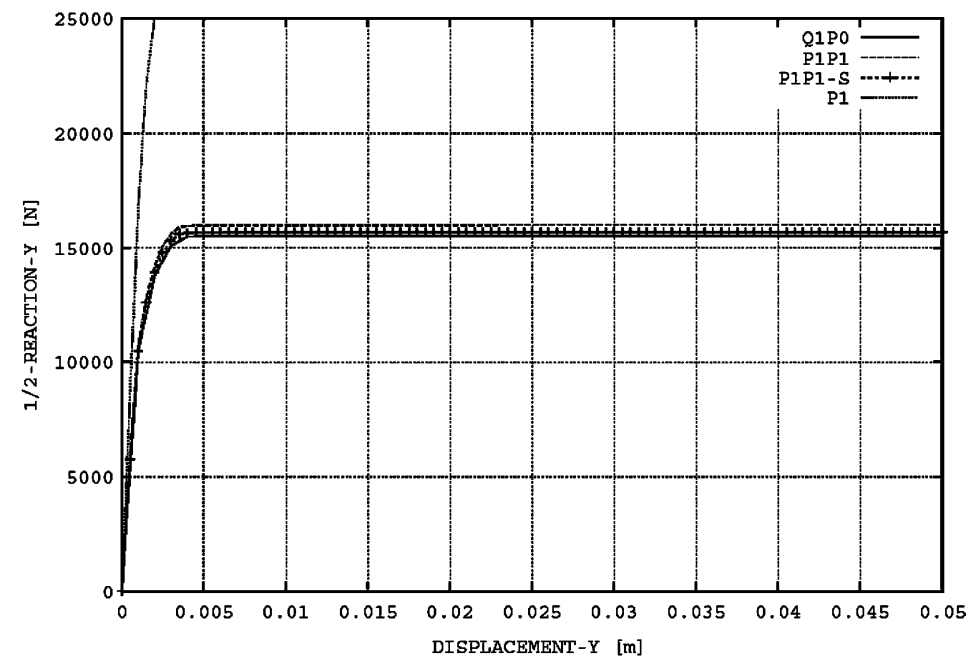

Fig. 9. (Half)-load-displacement curve for 2D Prandtl's punch test.

Fig. 9 shows (half)-load versus (point of application)-displacement curves for the $P 1, P 1 P 1, P 1 P 1-S$ and $Q 1 P 0$ cases. The results confirm the observations of the previous examples: the irreductible formulation locks almost completely, while the other three formulations yield practically overlapping curves. The CPU ratios obtained are 1.26/1.53/1.62/1.00.

Fig. 10 portrays contours of the effective plastic strain once the plastic flow is fully developed and the collapse mechanism can be appreciated (vertical displacement of the load $\delta=0.05 \mathrm{~m}$ ). The ability of the

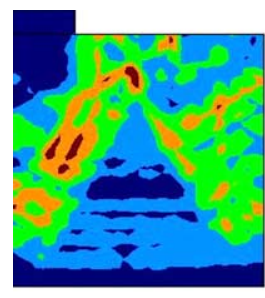

(a)

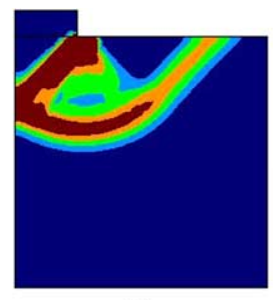

(b)

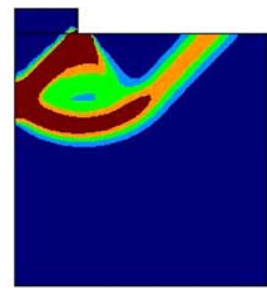

(c)

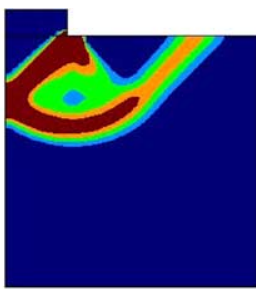

(d)

Fig. 10. Equivalent plastic strain contour fills for the 2D Prandtl's test with different formulations: (a) P1, (b) P1P1, (c) P1P1-S, (d) $Q 1 P 0$.

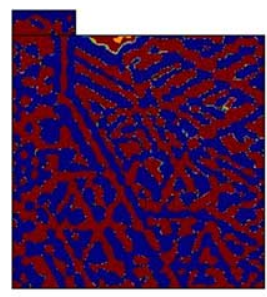

(a)

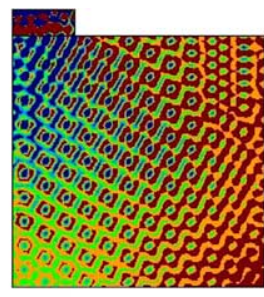

(b)

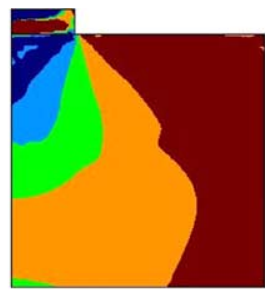

(c)

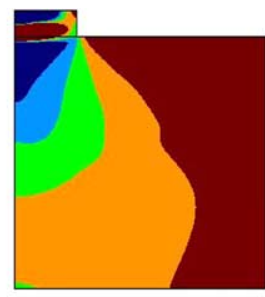

(d)

Fig. 11. Pressure contour fills for the 2D Prandtl's test with different formulations: (a) P1, (b) P1P1, (c) P1P1-S, (d) Q1P0. 
proposed formulation to capture the correct collapse mechanism and to match the performance of the mixed quadrilateral is again demonstrated.

Finally, Fig. 11 shows pressure contours at the same time of the deformation process, where the serious deficiencies of the standard formulations and the huge improvement achieved with a proper stabilization are evident.

\section{Conclusions}

This paper presents the formulation of stable mixed linear/linear simplicial elements (triangles and tetrahedrons) for incompressible elasticity and plasticity. The proposed stabilization is based on the orthogonal sub-grid scales approach and it circumvents the strictness of the BB-condition. The final method, consisting of coupling the standard algorithm for mixed elements with the explicit solution of the projection of the pressure gradient, yields an accurate and robust scheme, suitable for engineering applications in $2 \mathrm{D}$ and $3 \mathrm{D}$. Numerical examples show that results are free of volumetric locking and pressure oscillations, and qualitatively comparable to those obtained with the mixed Q1PO quadrilateral and hexahedron. It is remarkable that correct failure mechanisms with localized patterns of plastic deformation are obtained which show no influence of the mesh directional bias.

\section{Acknowledgements}

The authors gratefully acknowledge the inestimable help of Prof. R. Codina, in the form of unfailing suggestions and discussions.

\section{References}

[1] J.C. Simo, R.L. Taylor, K.S. Pister, Variational and projection methods for the volume constraint in finite deformation elastoplasticity, Comput. Methods Appl. Mech. Engrg. 51 (1985) 177-208.

[2] J.C. Simo, M.S. Rifai, A class of mixed assumed strain methods and the method of incompatible modes, Int. J. Numer. Methods. Engrg. 29 (1990) 1595-1638.

[3] C. Miehe, Aspects of the formulation and finite element implementation of large strain isotropic elasticity, Int. J. Numer. Methods Engrg. 37 (1994) 1981-2004.

[4] B.D. Reddy, J.C. Simo, Stability and convergence of a class of enhanced assumed strain methods, SIAM J. Numer. Anal. 32 (1995) 1705-1728.

[5] F. Brezzi, M. Fortin, Mixed and Hybrid Finite Element Methods, Springer, New York, 1991.

[6] D.N. Arnold, F. Brezzi, M.A. Fortin, Stable finite element for the Stokes equations, Calcolo 21 (1984) 337-344.

[7] J. Bonet, A.J. Burton, A simple average nodal pressure tetrahedral element for incompressible and nearly incompressible dynamic explicit applications, Commun. Numer. Methods Engrg. 14 (1998) 437-449.

[8] O.C. Zienkiewicz, J. Rojek, R.L. Taylor, M. Pastor, Triangles and tetrahedra in explicit dynamic codes for solids, Int. J. Numer. Methods Engrg. 43 (1998) 565-583.

[9] O. Klaas, A. Maniatty, M.S. Shephard, A stabilized mixed finite element method for finite elasticity. Formulation for linear displacement and pressure interpolation, Comput. Methods Appl. Mech. Engrg. 180 (1999) 65-79.

[10] R.L. Taylor, A mixed formulation for triangular and tetrahedral elements, in: R. Abascal, J. Domínguez, G. Bugeda (Eds.), Conference Proceedings on Métodos Numéricos en Ingeniería, SEMNI, Barcelona, Spain, 1999.

[11] C.R. Dohrmann, M.W. Heinstein, J. Jung, S.W. Key, W.R. Witkowsky, Node-based uniform strain elements for three-node triangular and four-node tetrahedral meshes, Int. J. Numer. Methods Engrg. 47 (2000) 1549-1568.

[12] R.L. Taylor, A mixed-enhanced formulation for tetrahedral elements, Int. J. Numer. Methods Engrg. 47 (2000) $205-227$.

[13] J. Bonet, H. Marriot, O. Hassan, An averaged nodal deformation gradient linear tetrahedral element for large strain explicit dynamic applications, Commun. Numer. Methods Engrg. 17 (2001) 551-561. 
[14] J. Bonet, H. Marriot, O. Hassan, Stability and comparison of different linear tetrahedral formulations for nearly incompressible explicit dynamic applications, Int. J. Numer. Methods Engrg. 50 (2001) 119-133.

[15] E. Oñate, J. Rojek, R.L. Taylor, O.C. Zienkiewicz, Linear triangles and tetrahedra for incompressible problem using a finite calculus formulation, in: Proceedings of European Conference on Computational Mechanics, ECCM, 2001.

[16] E.A. de Souza Neto, F.M.A. Pires, D.R.J. Owen, A new F-bar-method for linear triangles and tetrahedra in the finite strain analysis of nearly incompressible solids, in: Proceedings of VII International Conference on Computational Plasticity, Complas, 2003.

[17] T.J.R. Hughes, Multiscale phenomena: Green's function, Dirichlet-to-Neumann formulation, subgrid scale models, bubbles and the origins of stabilized formulations, Comput. Methods Appl. Mech. Engrg. 127 (1995) 387-401.

[18] T.J.R. Hughes, G.R. Feijoó, L. Mazzei, J.B. Quincy, The variational multiscale method—a paradigm for computational mechanics, Comput. Methods Appl. Mech. Engrg. 166 (1998) 3-28.

[19] R. Codina, J. Blasco, A finite element method for the Stokes problem allowing equal velocity-pressure interpolations, Comput. Methods Appl. Mech. Engrg. 143 (1997) 373-391.

[20] R. Codina, Stabilization of incompressibility and convection through orthogonal sub-scales in finite element methods, Comput. Methods Appl. Mech. Engrg. 190 (2000) 1579-1599.

[21] R. Codina, J. Blasco, Stabilized finite element method for transient Navier-Stokes equations based on pressure gradient projection, Comput. Methods Appl. Mech. Engrg. 182 (2000) 287-300.

[22] R. Codina, Stabilized finite element approximation of transient incompressible flows using orthogonal subscales, Comput. Methods Appl. Mech. Engrg. 191 (2002) 4295-4321.

[23] M. Chiumenti, Q. Valverde, C. Agelet de Saracibar, M. Cervera, A stabilized formulation for incompressible elasticity using linear displacement and pressure interpolations, Comput. Methods Appl. Mech. Engrg. 191 (2002) 5253-5264.

[24] M. Chiumenti, Q. Valverde, C. Agelet de Saracibar, M. Cervera, A stabilized formulation for incompressible plasticity using linear triangles and tetrahedra, Int. J. Plasticity (in press).

[25] C. Baiocchi, F. Brezzi, L. Franca, Virtual bubbles and Galerkin/least-squares type methods (Ga.L.S.), Comput. Methods Appl. Mech. Engrg. 105 (1993) 125-141.

[26] F. Brezzi, M.O. Bristeau, L. Franca, M. Mallet, G. Rogé, A relationship between stabilized finite element methods and the Galerkin method with bubble functions, Comput. Methods Appl. Mech. Engrg. 96 (1992) 117-129.

[27] T.J.R. Hughes, L.P. Franca, M. Balestra, A finite element formulation for computational fluid dynamics: V. Circumventing the Babuska-Brezzi condition: a stable Petrov-Galerkin formulation of the Stokes problem accomodating equal-order interpolations, Comput. Methods Appl. Mech. Engrg. 59 (1986) 85-99.

[28] T.J.R. Hughes, L.P. Franca, G.M. Hulbert, A new finite element formulation for computational fluid dynamics: VIII. The Galerkin/least-square method for advective-diffusive equations, Comput. Methods Appl. Mech. Engrg. 73 (1989) $173-189$.

[29] M. Cervera, C. Agelet de Saracibar, M. Chiumenti, COMET: COupled MEchanical and Thermal analysis, Data Input Manual, Version 5.0, Technical report IT-308, Available from <htpp://www.cimne.upc.es $>2002$.

[30] GiD: The personal pre and post preprocessor, Available from <htpp://www.gid.cimne.upc.es> 2002. 